\title{
Charged Fluid Dynamics in Scalar-Tensor Theories of Gravity with Torsion
}

\author{
Chih-Hung Wang ${ }^{* \dagger}$
}

November 4, 2018

\begin{abstract}
In scalar-tensor theories of gravity with torsion, the gravitational field is described in terms of a symmetric metric tensor $g$, a metriccompatible connection $\nabla$ with torsion, and a scalar field $\varphi$. The main aim is to explore an interaction of a charged perfect fluid and a scalar field $\varphi$ in a background electromagnetic and gravitational field described by $\{g, \nabla, \varphi\}$. The interaction is based on an action functional $S_{C}$ of a charged perfect fluid that is invariant under global conformal rescalings. Using a variational principle, we obtain equations of motion for the charged perfect fluid. Moreover, we verify that these equations of motion are equivalent to the gauge identities obtained from the invariance of an action functional under spacetime dffeomorphisms and a local $\mathrm{U}(1)$ gauge group.
\end{abstract}

\section{Action Functionals of Charged Perfect Fluids}

In Brans-Dicke theory [1] a direct interaction between the Brans-Dicke scalar field and matter is often said to violate the weak equivalence principle [7]. However, as Dirac [4] has shown one may consider gravity in a Weyl geometry which naturally induces such interactions. But little exploration has been made of gravitational scalar field interactions in a Cartan geometry. Dereli and Tucker [3] have noted that the motion of an electrically neutral spinless particle in the background geometry of Dirac's theory can also be reformulated in terms of an autoparallel of a Cartan geometry with torsion determined by the gradient of the Brans-Dicke scalar field [2]. In this note, we examine the motion of a relativistic charged fluid in a background Cartan geometry determined by a scalar field. We shall motivate a particular coupling of such a field to the fluid and derive the equations of motion from an action principle.

\footnotetext{
*Institute of Physics, Academia Sinica, Taipei 115, Taiwan, Republic of China(email : chhwang@phys.sinica.edu.tw)

${ }^{\dagger}$ Department of Physics, Lancaster University, UK (email : robbin1101@hotmail.com)
} 
In general relativity (GR), the theory of a relativistic perfect fluid with internal energy $\varepsilon(n)$, number density $n$, 4-velocity field $V$ composed of particles of rest mass $m$ and electric charge $q$ can be determined from the action

$$
\begin{aligned}
S_{G R} & =\int_{M} \Lambda_{G R}\left(e^{a}, A ; V, n, \lambda_{1}, \lambda_{2}\right) \\
& =\int_{M}\left(-m n(1+\varepsilon(n)) \star 1+q n A \wedge \star \widetilde{V}+\lambda_{1} \mathrm{~d}(n \star \widetilde{V})+\lambda_{2}(\widetilde{V} \wedge \star \widetilde{V}+\star 1)\right),
\end{aligned}
$$

where the background metric of spacetime is $g=e^{a} \otimes e_{a}$ and the background Maxwell field is $F=\mathrm{d} A$. Latin indices run over 0 to 3 . The signature is $(-,+,+,+)$ and $c=1$. The Lagrange multiplier fields $\lambda_{1}, \lambda_{2}$ ensure the conservation equation $\mathrm{d}(n \star \widetilde{V})=0$ and normalization of 4-velocity $g(V, V)=-1$. We use ; in $\Lambda_{G R}$ to separate background fields $e^{a}, A$ from the fluid's variables. $\widetilde{V}$ denotes the metric dual of $V$, i.e. $\widetilde{V} \equiv g(V,-)$, and $\star$ is the Hodge map. For any 1-form $\alpha, \widetilde{\alpha}=g^{-1}(\alpha,-)$, where $g^{-1}$ is the inverse of $g$. In a non-Riemannian Cartan background with specified metric $g$ and torsion forms $T^{a}$, we suppose that the gravitational scalar $\varphi$ couples to the relativistic fluid to modify the action to

$$
\begin{aligned}
S_{C} & =\int_{M} \Lambda_{C}\left(e^{a}, A, \varphi ; V, n, \lambda_{1}, \lambda_{2}\right) \\
& =\int_{M}\left(-\chi \varphi n(1+\varepsilon(n)) \star 1+q n A \wedge \star \widetilde{V}+\lambda_{1} \mathrm{~d}(n \star \widetilde{V})+\lambda_{2}(\widetilde{V} \wedge \star \widetilde{V}+\star 1)\right),
\end{aligned}
$$

where $\chi$ is a coupling constant. We have motivated the action by demanding that $\left.S_{C}\right|_{\varphi=\frac{m}{\chi}}=S_{G R}$ and that it be invariant under the following global rescalings

$$
\begin{array}{lcl}
\bar{e}^{a}=\Omega e^{a}, & \bar{A}=A, & \bar{\varphi}=\Omega^{-1} \varphi, \\
\bar{V}=\Omega^{-1} V, \quad \bar{n}=\Omega^{-3} n, & \bar{\varepsilon}(n)=\varepsilon, \quad \bar{\lambda}_{1}=\lambda_{1}, \quad \bar{\lambda}_{2}=\Omega^{-4} \lambda_{2},
\end{array}
$$

where $\Omega=$ constant. Here, the conformal weights of $V$ and $n$ are obtained by requiring that $\mathrm{d}(n \star \widetilde{V})=0$ and $g(V, V)=-1$ remain invariant under $\Omega$ transformations. Furthermore, we assume that $\chi$ and $q$ are also invariant under $\Omega$ transformations.

\section{Equations of Motion for the Charged Perfect Fluid}

By varying $S_{C}$ with respect to $V, n, \lambda_{1}$, and $\lambda_{2}$, we obtain

$$
\begin{aligned}
& q n \star A-n \star \mathrm{d} \lambda_{1}+2 \lambda_{2} \star \widetilde{V}=0, \\
& -\chi \varphi f \star 1+q A \wedge \star \widetilde{V}-\mathrm{d} \lambda_{1} \wedge \star \widetilde{V}=0, \\
& \mathrm{~d}(n \star \widetilde{V})=0, \\
& \widetilde{V} \wedge \star \widetilde{V}+\star 1=0,
\end{aligned}
$$


where $f=1+\varepsilon(n)+n \frac{\mathrm{d} \varepsilon}{\mathrm{d} n}$ is the index of the fluid [5]. By solving for $\lambda_{1}$ and $\lambda_{2}$, we can derive the equations of motion for $V$ and $n$ by substituting them into the rest of equations. There is no obvious way to know which two equations are best used for solving $\lambda_{1}$ and $\lambda_{2}$ since they both appear in the 3 -form equation (1). Using (1), (2) and (4), we can first solve for $\lambda_{2}$ to obtain $\lambda_{2}=\frac{1}{2} \chi \varphi n f$. Then, we eliminate $\lambda_{1}$ from (1) and take the interior derivative $\mathrm{i}_{V}$ to get

$$
q \mathrm{i}_{V} F+\mathrm{i}_{V} \mathrm{~d}(\chi \varphi f \widetilde{V})=0,
$$

where the solution of $\lambda_{2}$ has been used and $F=\mathrm{d} A$. Because of the identity $\mathrm{i}_{V} \mathrm{i}_{V} \theta \equiv 0$ for any p-form $\theta$, one recognizes that the 1 -form equation (5) has only 3 independent scalar equations. We consider the 3 independent equations (5) with (3) and (4) as equations of motion for $V$ and $n$. It is interesting to notice that equations (5), (3) and (4) do not involve any direct connection couplings. We can express (5) in terms of the torsion free Levi-Civita connection $\hat{\nabla}$ as

$$
\chi \varphi n f \hat{\nabla}_{V} V+q n \widetilde{\mathrm{i}_{V} F}+\chi \varphi \Pi_{V} \widetilde{\hat{\nabla} p}+\chi n f \Pi_{V} \widetilde{\hat{\nabla} \varphi}=0,
$$

where $p=n^{2} \frac{\mathrm{d} \varepsilon}{\mathrm{d} n}$ is the pressure per mass and $\Pi_{V}$ is a projection operator defined by $\Pi_{V} \equiv \mathbf{1}+V \otimes \widetilde{V}$. From (6), we find that $\varphi$ has a similar effect as the pressure per mass $p$ acting on the motion of the fluid. Moreover, a neutral dust-like $(q=p=0)$ fluid does not follow geodesic flow due to the scalar field effect. If we consider the background torsion field $T^{a}=e^{a} \wedge \frac{\mathrm{d} \varphi}{\varphi}[2]$, (6) becomes

$$
n \chi \varphi f \nabla_{V} V+q n \widetilde{\mathrm{i}_{V} F}+\chi \varphi \Pi_{V} \widetilde{\hat{\nabla} p}=0,
$$

where the non-Riemannian connection involves $\varphi$. From (7), it follows that the integral curves of $V$ for a neutral dust-like fluid are autoparallels of $\nabla$ rather than $\hat{\nabla}$.

Invariance of $S_{C}$ under spacetime diffeomorphism, local $\mathrm{SO}(3,1)$, and $\mathrm{U}(1)$ gauge transformations yield the gauge identities [6]

$$
\begin{aligned}
& \mathrm{D} \tau_{a}+\tau_{c} \wedge \mathrm{i}_{X_{a}} T^{c}+S_{b}{ }^{c} \wedge \mathrm{i}_{X_{a}} \mathrm{R}^{b}{ }_{c}+j \wedge \mathrm{i}_{X_{a}} F-\rho\left(\mathrm{i}_{X_{a}} \mathrm{~d} \varphi\right) \equiv 0, \\
& \mathrm{D} S_{a}{ }^{b}-\frac{1}{2}\left(\tau_{a} \wedge e^{b}-\tau^{b} \wedge e_{a}\right) \equiv 0, \\
& \mathrm{~d} j \equiv 0
\end{aligned}
$$

where 3 -forms $\tau_{a}, S_{a}{ }^{b}, j$, and 4 -form $\rho$ can be recognized as source currents of $e^{a}$, connection 1-forms $\omega^{a}{ }_{b}$ with torsion, $F$, and $\varphi$ respectively. D is a covariant exterior derivative with respect to $\omega^{a}{ }_{b}$ and $\mathrm{R}_{c}^{b}$ are the associated curvature 2-forms. From $S_{C}$, these source currents become

$$
\begin{aligned}
\tau_{a} & =\chi \varphi\left(V_{a}(n(1+\varepsilon)+p) \star \widetilde{V}+p \star e_{a}\right), \\
j & =q n \star V, \\
\rho & =-\chi n(1+\varepsilon) \star 1,
\end{aligned}
$$


and $S_{a}{ }^{b}=0$. [6] By substituting these source currents into (8), (9) and (10), we find that (3) is equivalent to (10) and using the first structure equation

$$
T^{a}=\mathrm{d} e^{a}+\omega^{a}{ }_{c} \wedge e^{c},
$$

and (4), Eq. (5) becomes Eq. (8). Eq. (9) is automatically satisfied.

I am very grateful to Robin Tucker and David Burton for helpful discussions during my PhD study and to Faculty of Science and Technology and Physics Department at Lancaster University for a travel grant to attend MG11.

\section{References}

[1] Brans, C. \& Dicke, R. H., Phys. Rev. 124, 925, (1961).

[2] Dereli, T. \& Tucker, R. W., Phys. Letts. B110, 206, (1982).

[3] Dereli, T. \& Tucker, R. W., Mod. Phys. Lett. A 17, 421-428, (2002).

[4] Dirac, P. A. M., Proc. Roy. Soc. Lond. A 333, 419, (1973).

[5] Lichnerowicz, A. Relativisitic Hydrodynamics and Magnetohydrodynamics (Benjamin, New York, 1967).

[6] Wang, C. H., Ph.D. Thesis, Lancaster, 2006 (unpublished).

[7] Will, C. M. Living Rev. Relativ. 9, 3, (2006). 